\title{
The Behavior of 2:1 and 3:1 Electrolytes at Polarizable Interfaces
}

\author{
Tímea Nagy ${ }^{1}$, Mónika Valiskó ${ }^{1}$, Douglas Henderson*2, and Dezső Boda ${ }^{1,2}$ \\ ${ }^{1}$ Department of Physical Chemistry, University of Pannonia, P.O. Box 158, H-8201 \\ Veszprém, Hungary \\ ${ }^{2}$ Department of Chemistry and Biochemistry, Brigham Young University, Provo UT 84602
}

December 1, 2010

\begin{abstract}
The behavior of the electrical double layer (DL) is known to be different at polarizable interfaces, specifically, at a metallic electrode (where the dielectric constant of the electrode is infinitely large, $\epsilon_{1} \rightarrow \infty$ ) and at an air/electrolyte interface (where the dielectric constant of the electrode is $\epsilon_{1}=1$ ) than is the case for unpolarized interfaces. For the polarized interface, if multivalent ions are present, these ions are attracted/repelled more than is the case for monovalent ions. Therefore, the divalent/trivalent ions (assumed to be cations to be specific) accumulate near the metallic electrode more than for the unpolarized electrode and a charge inversion occurs. In such asymmetric electrolytes, this results in a large potential at zero electrode charge. The behavior is reversed for the air/electrolyte interface. This is more pronounced at low reduced temperatures (or, equivalently, at high ionic couplings). The anomalous capacitance behavior of the DL is seen for the unpolarized electrode, where the temperature derivative of the capacitance is positive at low reduced temperatures (characteristic of electrolytes with ions with high ionic couplings or molten salt DLs at room temperatures) while it is negative at high reduced temperatures (characteristic or aqueous solutions of monovalent salts at room temperatures). At least for the states we consider, this anomalous behavior is enhanced for the
\end{abstract}

* Author for correspondence 
air/electrolyte interface but vanishes for a metallic electrode. Our Monte Carlo simulations of these phenomena are reported.

\section{Introduction}

The authors are pleased to dedicate this article to John Prausnitz in recognition of his many contributions to the theory of fluids.

An electrical double layer (DL) is formed when charged particles (say the ions in an electrolyte) are near a charged electrode. The term DL is based on the intuitive idea that the charge of the electrode and the excess charge in the electrolyte form two layers. In reality, the situation can be more complex with layering and even charge reversal within one or both the electrode and electrolyte layers. Thus, the electrode and electrolyte excess charges are layers only in an average sense. We will use the term DL and layers in this average sense. In any case, there is a potential difference between the two layers; these layers form a capacitor with a capacitance. Experiments typically measure this capacitance as a function of the electrode charge or potential. A more detailed probe of the nature of the DL would be the microscopic structure of the DL that is described by the density and charge profiles. This has not been measured directly in an experiment but can be obtained from theory or simulation.

Most simulations and theories are based on what is called the primitive model (PM) of the electrolyte, in which the solvent is represented by a dielectric continuum and the ions are represented by charged hard spheres. If the further assumption or restriction that the hard sphere ions all have the same diameter, $d$, is made, the model is called the restricted primitive model (RPM). Further, a primitive model of the electrode is usually assumed, where the electrode is a charged hard smooth plane with all the electrode charge located on the surface of the plane (zero skin depth). It is certainly possible to formulate more sophisticated models of the electrolyte and electrode but this is rarely done because of the additional computational complexity. For example, including explicit solvent molecules restricts a simulation to high concentrations because a very large number of solvent molecules would be required at concentrations much below about $1 \mathrm{M}$ in order that there is a reasonable number of ions and that reliable averages can be obtained. 
The conventional theory of the DL is the Poisson-Boltzmann theory of Gouy, Chapman, and Stern $^{1-3}$ (GCS). This is based on the PM but employs two further assumptions. Correlations among the ions (except those provided directly by the interaction with the mean field) are neglected and the size of the ions is neglected, other than assuming a nonzero distance of closest approach (or Stern layer) between the ions and the electrode. The GCS theory suffers from at least four defects. The GCS charge profiles are monotonic whereas simulations show that they can be nonmonotonic when multivalent ions are present or the coupling is strong. Further, a good theory would give the same result, or at least a close result, no matter whether it is the electrode charge or potential that is specified. This is not the case for the GCS theory. The superficial observation is often made that the GCS density profiles are in good agreement with simulation results for monovalent electrolytes. However, this is true only if the electrode charge density is chosen as the independent variable. In an experiment, it is the electrode potential that is commonly used. The GCS charge profiles are in poor agreement with simulation results, even for monovalent ions, if the electrode potential is specified. ${ }^{4}$ For multivalent ions, the GCS profiles are poor, independently of whether the electrode charge or potential is fixed. Additionally, at lower temperatures (say frozen electrolytes), problems are apparent in the GCS theory even when applied to monovalent ions. ${ }^{5,6}$ The third problem with the GCS theory is that for a symmetric electrolyte the coion profile is assumed to be the reciprocal of the counterion profile. It has been known for a while (discussed recently ${ }^{7}$ ) that this is not the case. Finally in the GCS theory, the ions can accumulate in unlimited numbers near the electrode. Recent simulations have shown that the capacitance tends to zero at high electrode charge. ${ }^{8}$ This is due to the well-known fact that there is a limit to how many counterions can be accomodated near the electrode. The GCS theory exhibits the opposite behavior.

The modified Poisson-Boltzmann (MPB) theory of Outhwaite and Bhuiyan ${ }^{9}$ and density functional theory (DFT) ${ }^{10-14}$ seem to be reasonably satisfactory but should be tested further to verify that they are useful for a specific application. Recent developments made DFT appropriate to study inhomogeneous electrolytes at low temperatures ${ }^{15,16}$ and in systems with large concentration variations.${ }^{17,18}$ The extension of the theory to three dimensions is in progress. ${ }^{19}$ The more easily 
applied mean spherical approximation ${ }^{20}$ is fairly good for monovalent and divalent ions at room temperature but fails under more extreme situations and, in any case, is a linear response theory and can be applied only for very small electrode charge.

There have been several previous simulations of the DL for polarized and unpolarized electrodes and for symmetric and asymmetric electrolytes. The earliest simulations of the DL were those of Torrie, Valleau, Patey, and Outhwaite, ${ }^{21-28}$ followed by Snook and van Megen. ${ }^{29,30}$ After a long hiatus, new simulations of the DL have been performed recently by ourselves ${ }^{5-7,31-36}$ to study the question of whether the counterion and coion profiles are in symmetric electrolytes are reciprocals, ${ }^{7}$ contact value theorems, ${ }^{34-37}$ highly charged electrodes,${ }^{38,39}$ and high density charged hard spheres ${ }^{40}$ as a model for DLs with ionic liquids.

In this study, we consider polarizable interfaces where the boundary of the electrolyte and the "electrode" separates two regions with different dielectric constants. The term "electrode" is used in a general sense: it can be a metal (when its dielectric constant $\epsilon_{1} \rightarrow \infty$ ), air $\left(\epsilon_{1}=1\right.$ ), or various other objects such as a biological membrane, a macromolecule, a colloid particle, etc. In the presence of a polarizable interface, the interactions are no longer pair-wise additive which makes the theoretical study of such systems difficult. ${ }^{9,23,41-51}$ Simulations, on the other hand, can be applied to polarizable interfaces straightforwardly. The calculation of the interaction with the polarization charge is a challenge, ${ }^{52,53}$ but when the interface is a single infinite flat wall, the interactions can be handled with the image charge method (see section 2). Although several simulation studies have been published using image charges ${ }^{23,27,48,54-63}$ the number of such studies is much smaller than those for the unpolarizable interface. A polarizable electrode with a layer with a dielectric constant that differed from that of both the electrode and the electrolyte has been studied by simulation. ${ }^{45}$

The capacitance anomaly at low reduced temperatures means that the capacitance of the DL increases with decreasing temperature (in agreement with simple theories), have a maximum, and decreases at low temperatures. ${ }^{5,6}$ Only more developed theories were able to reproduce this behavior. ${ }^{16,34,64-66}$

We have calculated the anomalous capacitance behavior for 2:1 and 3:1 electrolytes and found a behavior similar to the 1:1 case if the reduced temperature is scaled appropriately. ${ }^{31}$ Alawneh et 
al. ${ }^{59}$ have studied the capacitance anomaly for a symmetric 1:1 0.1 M electrolyte near a polarizable electrode. They found that the capacitance anomaly was enhanced for the air/electrolyte interface but vanishes if the electrode is metallic. This is in agreement with the results of Outhwaite and Bhuiyan $^{49}$ who used a modified Poisson-Boltzmann theory with point charges. In this paper, we connect these two lines of studies and report our Monte Carlo results for 2:1 and 3:1 electrolytes near polarizable interfaces.

\section{Model}

In this simulation study, the electrode is considered to be uniform and the dielectric coefficient of the electrode is $\epsilon_{1}$. The solvent is treated as a dielectric continuum whose dielectric coefficient is $\epsilon_{2}=78.5$. The ions are charged hard spheres whose diameter is $d$. The charge of an ion of species $i$ is $q_{i}=z_{i} e$, where $z_{i}$ is the valence and $e$ is the elementary charge. The specific cases of 2:1 and 3:1 electrolytes are examined here. The electrode has a uniform surface charge density, $\sigma$.

When the dielectric constant of the solvent and electrode differ, polarization charges are induced on the electrode by the ions. The potential of these polarization charges is equal to the potential of an image charge located in an appropriate position. Let us assume a point charge $q_{i}$ located at a point $\mathbf{r}_{i}=\left(x_{i}, y_{i}, z_{i}\right)$ in the $\epsilon_{2}$ region $\left(z_{i}>0\right)$, where the dielectric boundary is at $z=0$. The image charge of this charge has a magnitude $q^{\prime}=\theta q$ and is located at $\mathbf{r}_{i}^{\prime}=\left(x_{i}, y_{i},-z_{i}\right)$, where

$$
\theta=\frac{\epsilon_{1}-\epsilon_{2}}{\epsilon_{1}+\epsilon_{2}}
$$

The potential produced by the charge and its image charge at a position $\mathbf{R}$ in the electrolyte $(z>0)$ is

$$
\phi_{i}(\mathrm{R})=\frac{1}{4 \pi \epsilon_{0} \epsilon_{2}}\left(\frac{q_{i}}{\left|\mathbf{r}_{i}-\mathbf{R}\right|}+\frac{\theta q_{i}}{\left|\mathbf{r}_{i}^{\prime}-\mathbf{R}\right|}\right)
$$

where $\epsilon_{0}$ is the permittivity of free space. The total electrostatic energy between ion pairs then is computed as

$$
U_{\text {pair }}=\frac{1}{2} \sum_{i} \sum_{j \neq i} q_{j} \phi_{i}\left(\mathbf{r}_{j}\right) .
$$


This energy becomes infinite if any of the ion-pairs overlaps. The energy corresponding to the interactions of the ions with their own image charges is

$$
U_{\text {self }}=\frac{1}{8 \pi \epsilon_{0} \epsilon_{2}} \sum_{i} \frac{\theta q_{i}^{2}}{2 z_{i}} .
$$

The interaction of a charge with the electrode charge is

$$
U_{\mathrm{wall}}=-\sum_{i} \frac{(1+\theta) q_{i} \sigma}{2 \epsilon_{0} \epsilon_{2}} z_{i}
$$

Again, this energy becomes infinite if a hard sphere ion overlaps with the electrode.

The cases $\epsilon_{1}=1$ and $\epsilon_{1} \rightarrow \infty$ correspond to $\theta=0.975$ and $\theta=-1$, respectively. The first case corresponds to an air/electrolyte, while the second case corresponds to a metal/electrolyte interface. At first thought, one might think that $\epsilon_{2}=78.5$, the value for water, is large enough to be well on its way to $\infty$. However, this case corresponds to $\theta=0$ and is almost midway between the other two cases that we consider.

The electrostatic images are not real, just as the visual image seen in a mirror is not real. There is no world behind the mirror. It is the induced charge that is real and this resides on the surface of the electrode. However, the electrostatic potential of the images is identical to that of the induced charges.

\section{Monte Carlo Simulations}

We have performed canonical MC simulations, where the temperature $T=300 \mathrm{~K}$, the numbers of the various ionic species $N_{i}$, and the volume of the simulation cell was fixed. The simulation cell was placed between two hard walls, where the left hand side wall is the dielectric interface carrying the $\sigma$ surface charge, while the right hand side wall is uncharged. The typical number of cations in a simulation was 200 , while the number of anions was chosen so that the simulation cell

is charge neutral. In a simulation, $2 \times 10^{6}$ cycles were performed, where in one cycle all particles were attempted to move. Metropolis sampling was applied with the usual particle displacements 
(50\% of the moves were a small move with respect to the old position, while the other $50 \%$ of the moves were - possibly long - jumps to randomly selected positions in the simulation cell). In the lateral dimensions periodic boundary conditions were applied. To handle the effect of ions in the periodic cells, the charged sheet method - originally introduced by Torrie and Valleau ${ }^{21,22}$ and developed further by Boda et al. ${ }^{67}$ - was used.

The results of the simulations are the density profiles $\rho_{i}(z)$. From the density profiles the charge profile is calculated as

$$
q(z)=\sum z_{i} \rho_{i}(z)
$$

To compute the potential profile from the charge profile, Poisson's equation has to be solved with appropriate boundary conditions. Here we use a method (developed recently ${ }^{68}$ ), in which Neumann boundary conditions are used. The resulting equation is

$$
\Phi(z)=-\frac{1}{\epsilon_{0}} \int_{-\infty}^{z}\left[\int_{-\infty}^{z^{\prime}} q\left(z^{\prime \prime}\right) d z^{\prime \prime}\right] d z^{\prime}-\frac{1}{\epsilon_{0}} \sigma z+C_{2}
$$

where the first integration constant is set by the Neumann boundary condition that the normal electric field is zero outside the simulation cell and the second integration constant $C_{2}$ can be chosen arbitrarily. Here, we set the zero level of the potential to the central bulk region of the cell.

In this work, we use reduced units. The density profiles are plotted normalized by their bulk values: $g_{i}(z)=\rho_{i}(z) / \rho_{i}^{\mathrm{B}}$. The bulk density was chosen to yield $0.1 \mathrm{M}$ salt concentration for $d=4.25$ $\AA$. The reduced temperature is $T^{*}=4 \pi \epsilon_{0} \epsilon_{2} d k T / e^{2}$, the reduced surface charge is $\sigma^{*}=\sigma d^{2} / e$, and the reduced potential is $\Phi^{*}=4 \pi \epsilon_{0} \epsilon_{2} \Phi d / e$. Using these definitions, the reduced differential capacitance is defined as

$$
C^{*}=\left(\frac{d \sigma^{*}}{d \Phi^{*}}\right)_{\sigma^{*}=0}
$$

and computed from fitting to the results of 3-5 simulations performed for various small surface charges close to zero. 


\section{Results}

Density profiles, normalized to be unity, are shown in Figs. 1 and 2 for a 2:1 electrolyte at a low $\left(T^{*}=0.22\right)$ and high $\left(T^{*}=0.9\right)$ reduced temperatures at an uncharged electrode. Figure 1 is for the air/electrolyte interface $\left(\epsilon_{1}=1\right)$, while Fig. 2 is for the metallic electrode $\left(\epsilon_{1} \rightarrow \infty\right)$. For definiteness, the divalent ion is positive (black solid line) and the monovalent ion (red dashed line) is negative. Both ions are attracted or repelled by the metallic or air/electrolyte interface, respectively. The divalent ions are more strongly attracted or repelled than are the monovalent ions. This is because divalent ions induce four times larger polarization charge (the image charge is larger, see the self term in Eq. 4). For the metallic electrode, this induced charge is attractive, while for the air/electrolyte interface it is repulsive.

Charge inversion can be observed which is a straight consequence of the asymmetry in the ionic charges. ${ }^{33}$ This charge inversion results in a nonzero electrode potential at zero electrode charge (PZC) as shown in Fig. 3. The PZC potential is negative for $\epsilon_{1}=1$, while it is positive for $\epsilon_{1} \rightarrow \infty$.

The charge inversion and the nonzero PZC potential are present for the nonpolarizable electrode $\left(\epsilon_{1}=\epsilon_{2}\right)$ too, ${ }^{33}$ where it is a result of charge asymmetry only.

Electrode polarization influences this effect because there is an additional force that acts differently on cations and anions thus polarizing the DL. The PZC potential is more positive for lower reduced temperatures for the metallic electrode (bottom panel). In this case, electrode polarization enhances the effect of charge asymmetry. This is because the electrostatic correlations that produce this phenomenon are stronger at lower $T^{*}$. Stronger electrostatic correlations enhance both effects.

In the case of the air/electrolyte (top panel) the opposite behavior is observed. Again, stronger electrostatic correlations enhance both effects but now they act in the opposite directions. Seemingly, reducing the temperature has a stronger effect on charge asymmetry, thus the PZC potential becomes less negative as $T^{*}$ decreases.

The nonzero PZC potential is often taken as empirical evidence of specific adsorption (or chemisorption). We see here that it can arise from purely physical phenomena.

The potential profiles for a 2:1 electrolyte are plotted in Figs. 4 and 5 for a small positive and negative electrode charge density and an uncharged electrode. Figures 4 and 5 refer to reduced 
temperatures $T^{*}=0.9$ and $T^{*}=0.22$, respectively. For the air/electrolyte interface (top panels of Figs. 4 and 5), changing the surface charge only a little (from $\sigma^{*}=-0.0032$ to 0.0032 ) has a large effect on the potential: it actually becomes positive. The change in potential has similar magnitudes for the two temperatures, therefore the value of the capacitance is also similar in the two cases. This can be seen explicitly in Fig. 6, where the inverse capacitance is plotted as a function of $T^{*}$ for a 2:1 (left side) and a 3:1 (right side) electrolyte. The inverse capacitance is similar for $T^{*}=0.22$ and 0.9 (top-left panel) and there is a minimum in between (which corresponds to a maximum in $C^{*}$ as seen before). Comparing the behavior of the potential profiles for $\epsilon_{1}=1$, it is seen that the diffuse layer is much wider in the case of $T^{*}=0.22$. As discussed before, depletion of the density profiles at the interface due to stronger attraction between ions in the bulk phase than between ions and the electrode is characteristic in the temperature-regime, where the capacitance anomaly occurs.

In the case of the metallic electrode (bottom panels of Figs. 4 and 5), the situation is completely different. For large $T^{*}$ (Fig. 4), the small change in $\sigma^{*}$ results in a large change in $\Phi^{*}$, which corresponds to a small capacitance (or large $1 / C^{*}$ as seen in the bottom panels of Fig. 6). Decreasing the temperature (Fig. 5), the DL becomes wider and the potential becomes more positive. The small change in $\sigma^{*}$ now results in a small change in the potential that corresponds to a large capacitance (or small $1 / C^{*}$ as seen in the bottom panels of Fig. 6). An explanation of this behavior can be the following. The attractive induced charge enhanced by the low temperature produces a strong charge inversion in the DL (top panel of Fig. 2). This means that there is a well pronounced layer of divalent cations at the electrode and a well pronounced, but wider layer of anions in the adjacent region farther from the electrode. This results in a long-range dipole field as seen in the bottom panel of Fig. 5. It seems that a little change in the electrode charge influences mainly the inner cation layer. The effect of the surface charge is a "short-range" effect as seen in the bottom panel of Fig. 5 (magnified in the inset). Because of this, the anomalous temperature dependence of the capacitance vanishes for the metallic electrode.

The inverse capacitance can be written up as a sum of two capacitances, those due to the inner (or Helmholtz) layer and the diffuse layer (these two layers behave as capacitors connected 
in series):

$$
\frac{1}{C^{*}}=\frac{1}{C_{\mathrm{H}}^{*}}+\frac{1}{C_{\mathrm{D}}^{*}}=2 \pi+\frac{1}{C_{\mathrm{D}}^{*}}
$$

Figure 6 shows the diffuse layer capacitances too. Plotting this way has the advantage that $1 / C_{\mathrm{D}}^{*}$ changes continuously. In the case of the 3:1 electrolyte at the metallic electrode (bottom-right panel) the inverse diffuse layer capacitance becomes negative (so the capacitance diverges). This is probably due to the strong charge inversion at the metallic electrode. For a negative surface charge, for example, cations overcharge the electrode and from the point of view of the wide diffuse layer the electrode has an apparent positive charge. Important changes occur as an effect of changing $\sigma$ in the compact tightly bound inner layer as seen in the bottom panel of Fig. 5 .

Reduced units were used in this work. To connect the reported values to practical applications, we give some representative values in real units. The reduced surface charge $\sigma^{*}=0.0032$ corresponds to $\sigma=0.00284 \mathbf{C m}^{-2}$ for $d=4.25 \AA$. The reduced potential $\Phi^{*}=1$ corresponds to $\Phi=43.16 \mathrm{mV}$, while the reduced capacitance $C^{*}=1$ corresponds to $C=20.55 \mathbf{F m}^{-2}$ for $d=4.25 \AA$ and $\epsilon_{2}=78.5$. For these values of $d$ and $\epsilon_{2}$, the reduced temperatures $T^{*}=0.22$ and 0.9 correspond to the temperatures $T=110.2$ $\mathbf{K}$ and $450.8 \mathrm{~K}$, respectively. The corresponding values for $d=3 \AA$ are $T=156.1 \mathrm{~K}$ and 638.6 K. Alternatively, the small reduced temperature $T^{*}=0.22$ at room temperature $T=298.15 \mathrm{~K}$ corresponds to $\epsilon_{2}=29$ for $d=4.25 \AA$ and $\epsilon_{2}=41.1$ for $d=3 \AA$.

These values for the dielectric constant are far from being unrealistic even for an aqueous electrolyte. Based on the measurements of Barthel et al., ${ }^{69}$ Fawcett and Tikanen $^{70}$ suggested using the experimental dielectric constant of the solution instead of the dielectric constant of the pure solvent. The dielectric constant of concentrated electrolytes is smaller than that of dilute electrolytes due to dielectric saturation. For example, the dielectric constant of a $3 \mathrm{M} \mathrm{NaCl}$ solution is about 46.2, while that of a $2 \mathrm{M} \mathrm{CaCl}_{2}$ solution is 38.9. In a recent paper, Vincze et al. ${ }^{71}$ suggested using experimental data for the dielectric constant of the electrolyte together with the radii of "bare" ions (for example, the Pauling radii). They have shown that taking into account the explicit change in the solvation energy with increasing concentration, 
the nonmonotonic concentration dependence of the activity coefficient of electrolytes can be reproduced without the assumption of a "solvated" ion radius, such as the "historical" value $(d=4.25 \AA)$ used since the works of Torrie and Valleau. Vincze et al. proposed that using this enlarged, "solvated" ion radius is unphysical and unnecessary.

\section{Summary}

Our results extend the earlier study of Alawneh et al. ${ }^{59}$ who observed that the anomalous capacitance behavior vanishes at the metallic electrode. Our results for 2:1 and 3:1 electrolytes confirm their findings.

Our earlier results for 2:1 and 3:1 electrolytes near an unpolarizable electrode ${ }^{31}$ has been extended to polarizable electrodes. In the case of the air/electrolyte interface we find similar capacitance behavior as in the $\epsilon_{1}=\epsilon_{2}$ case. The maximum of the capacitance occurs at a larger temperature when a multivalent cation is present. As we have shown before, the $C^{*}$ vs. $T^{*}$ functions behave similarly if we scale the reduced temperature as $T^{*} /\left(z_{+} z_{-}\right) .{ }^{31}$ The same behavior is also observed here (see Fig. 6).

In the case of the metallic electrode, an interesting phenomenon is observed for 2:1 and 3:1 electrolytes. The strong attraction of induced charges at low reduced temperatures produces a compact dipole layer of cations and anions at the electrode. This results in a very wide diffuse layer with properties uninfluenced by the surface charge (large capacitance), while changing the surface charge has its major effect in the dipole layer close to the electrode.

\section{Acknowledgements}

These simulations were made possible by a generous allotment of computer time by the Ira and Mary Lou Fulton supercomputer facilty at Brigham Young University. This is acknowledged with thanks. The work was supported by the Hungarian National Research Fund (OTKA K75132 and F68641) and the János Bolyai Research Fellowship. 


\section{References}

[1] Gouy, G. Sur la constitution de la charge electrique a la surface d'un electrolyte. J. Phys. (Paris) 1910. 9, 457.

[2] Chapman, D.L. A contribution to the theory of electrocapillarity. Phil. Mag. 1913. 25, 475.

[3] Stern, O. Zur theorie der elektrolytischen doppelschicht. Zeit. Elektrochemie 1924. 30, 508.

[4] Henderson, D.; Boda, D. Insights from theory and simulation on the electrical double layer. Phys. Chem. Chem. Phys. 2009. 11, 3822.

[5] Boda, D.; Henderson, D.; Chan, K.Y. Monte Carlo study of the capacitance of the double layer in a model molten salt. J. Chem. Phys. 1999. 110, 5346.

[6] Boda, D.; Henderson, D.; Chan, K.Y.; Wasan, D.T. Low temperature anomalies in the properties of the electrochemical interface. Chem. Phys. Lett. 1999. 308, 473.

[7] Bhuiyan, L.B.; Outhwaite, C.W.; Henderson, D. Some simulation and modified PoissonBoltzmann theory results for the contact values of an electrolyte near a charged electrode. $J$. Electroanal. Chem. 2007. 607, 54.

[8] Lamperski, S.; Henderson, D. Simulation study of capacitance of the electrical double layer of an electrolyte near a highly charged electrode. Mol. Sim. 2010.

[9] Outhwaite, C.W.; Bhuiyan, L.B. An improved modified Poisson-Boltzmann equation in electric-double-layer theory. J. Chem. Soc. Faraday. Trans. II. 1983. 79, 707.

[10] Rosenfeld, Y. Free-energy model for the inhomogeneous hard-sphere fluid mixture and densityfunctional theory of freezing. Phys. Rev. Lett. 1989. 63, 980.

[11] Kierlik, E.; Rosinberg, M.L. Free-energy density functional for the inhomogeneous hard-sphere fluid - application to interfacial adsorption. Phys. Rev. A 1990. 42, 3382.

[12] Kierlik, E.; Rosinberg, M.L. Density-functional theory for inhomogeneous fluids - adsorption of binary-mixtures. Phys. Rev. A 1991. 44, 5025. 
[13] y Teran, L.M.; Suh, S.; White, H.S.; Davis, H. A nonlocal free-energy density-functional approximation for the electrical double-layer. J. Chem. Phys. 1990. 92, 5087.

[14] Rosenfeld, Y.; Schmidt, M.; Lowen, H.; Tarazona, P. Fundamental-measure free-energy density functional for hard spheres: Dimensional crossover and freezing. Phys. Rev. E 1997. 55, 4245.

[15] Pizio, O.; Patrykiejew, A.; Sokołowski, S. Phase behavior of ionic fluids in slitlike pores: A density functional approach for the restricted primitive model. J. Chem. Phys. 2004. 121, 11957.

[16] Reszko-Zygmunt, J.; Sokołowski, S.; Henderson, D.; Boda, D. Temperature dependence of the double layer capacitance for the restricted primitive model of an electrolyte solution from a density functional approach. J. Chem. Phys. 2005. 122, 084504.

[17] Gillespie, D.; Nonner, W.; Eisenberg, R.S. Coupling Poisson-Nernst-Planck and density functional theory to calculate ion flux. J. Phys.: Cond. Matt. 2002. 14, 12129.

[18] Gillespie, D.; Nonner, W.; Eisenberg, R.S. Density functional theory of charged, hard-sphere fluids. Phys. Rev. E 2003. 68, 031503.

[19] Knepley, M.G.; Karpeev, D.A.; Davidovits, S.; Eisenberg, R.S.; Gillespie, D. An efficient algorithm for classical density functional theory in three dimensions: Ionic solutions. J. Chem. Phys. 2010. 132, 124101.

[20] Blum, L. Theory of electrified interfaces. J. Phys. Chem. 1977. 81, 136.

[21] Torrie, G.M.; Valleau, J.P. Monte-Carlo study of an electrical double-layer. Chem. Phys. Lett. 1979. 65,343 .

[22] Torrie, G.M.; Valleau, J.P. Electrical double-layers 1. Monte Carlo study of a uniformly charged surface. J. Chem. Phys. 1980. 73, 5807.

[23] Torrie, G.M.; Valleau, J.P.; Patey, G.N. Electrical double-layers 2. Monte-Carlo and HNC studies of image effects. J. Chem. Phys. 1982. 76, 4615. 
[24] Valleau, J.P.; Torrie, G.M. The electrical double-layer 3. Modified Gouy-Chapman theory with unequal ion sizes. J. Chem. Phys. 1982. 76, 4623.

[25] Torrie, G.M.; Valleau, J.P. Electrical double-layers 4. Limitations of the Gouy-Chapman theory. J. Phys. Chem. 1982. 86, 3251.

[26] Valleau, J.P.; Torrie, G.M. Electrical double-layers 5. Asymmetric ion wall interactions. J. Chem. Phys. 1984. 81, 6291.

[27] Torrie, G.M.; Valleau, J.P.; Outhwaite, C.W. Electrical double-layers 6. Image effects for divalent ions. J. Chem. Phys. 1984. 81, 6296.

[28] Carnie, S.L.; Torrie, G.M.; Valleau, J.P. Ion-size effects in the primitive model double-layer. Mol. Phys. 1984. 53, 253.

[29] van Megen, W.; Snook, I. The grand canonical ensemble Monte-Carlo method applied to the electrical double-layer. J. Chem. Phys. 1980. 73, 4656.

[30] Snook, I.; van Megen, W. Finite ion size effects in the electrical double-layer - a Monte-Carlo study. J. Chem. Phys. 1981. 75, 4104.

[31] Valiskó, M.; Henderson, D.; Boda, D. The capacitance of the electrical double layer of valenceasymmetric salts at low reduced temperatures. J. Mol. Liquids 2007. 131-132, 179.

[32] Boda, D.; Fawcett, W.R.; Henderson, D.; Sokołowski, S. Monte Carlo, density functional theory, and Poisson-Boltzmann theory study of the structure of an electrolyte near an electrode. J. Chem. Phys. 2002. 116, 7170.

[33] Valiskó, M.; Henderson, D.; Boda, D. Competition between the effects of asymmetries in ion diameters and charges in an electrical double layer studied by Monte Carlo simulations and theories. J. Phys. Chem. B 2004. 108, 16548.

[34] Henderson, D.; Boda, D. On a conjecture of Fawcett. J. Electroanalytical Chem. 2005. 582, 16. 
[35] Henderson, D.; Bhuiyan, L.B. Evidence for a second contact value formula for the electric double layer. Mol. Sim. 2007. 33, 953.

[36] Henderson, D.; Bhuiyan, L.B. Simple expressions for contact values of density profiles in a planar double layer. J. Chem. Theor. Comp. 2009. 5, 1985.

[37] Bhuiyan, L.B.; Outhwaite, C.W.; Henderson, D. Evidence from Monte Carlo simulations for a second contact value theorem for a double layer formed by 2:1/1:2 salts at low electrode charges. Mol. Phys. 2009. 10\%, 343.

[38] Valiskó, M.; Gillespie, D.; Boda, D. Selective adsorption of ions with different diameter and valence at highly-charged interfaces. J. Phys. Chem. C 2007. 111, 15575.

[39] Lamperski, S.; Outhwaite, C.W.; Bhuiyan, L.B. The electric double-layer differential capacitance at and near zero surface charge for a restricted primitive model electrolyte. J. Phys. Chem. B 2009. 113, 8925 .

[40] Lamperski, S.; Bhuiyan, L.B. Counterion layering at high surface charge in an electric double layer. Effect of local concentration approximation. J. Electroanal. Chem. 2003. 540, 79.

[41] Carnie, S.L.; Chan, D.Y.C. Correlations in inhomogeneous Coulomb systems. Mol. Phys. 1984. $51,1047$.

[42] Kjellander, R.; Marčelja, S. Inhomogeneous Coulomb fluids with image interactions between planar surfaces. i. J. Chem. Phys. 1985. 82, 2122.

[43] Vertenstein, M.; Ronis, D. A theory of electrolyte solutions near a polarizable surface. $J$. Chem. Phys. 1987. 87, 4132.

[44] Attard, P.; Mitchell, D.J.; Ninham, B.W. Beyond Poisson-Boltzmann: Images and correlations in the electric double layer. II. Symmetric electrolyte. J. Chem. Phys. 1988. 89, 4358.

[45] Henderson, D.; Gillespie, D.; Nagy, T.; Boda, D. Monte Carlo simulation of the electric double layer: dielectric boundaries and the effects of induced charge. Mol. Phys. 2005. 103, 2851. 
[46] Wernersson, E.; Kjellander, R. On the effect of image charges and ion-wall dispersion forces on electric double layer interactions. J. Chem. Phys. 2006. 125, 154702.

[47] Wernersson, E.; Kjellander, R. Image charges and dispersion forces in electric double layers: The dependence of wall-wall interactions on salt concentration and surface charge density. $J$. Phys. Chem. B 2007. 111, 14279.

[48] Bhuiyan, L.B.; Outhwaite, C.W.; Henderson, D.; Alawneh, M. A modified Poisson-Boltzmann theory and Monte Carlo simulation study of surface polarization effects in the planar diffuse double layer. Mol. Phys. 2007. 105, 1395.

[49] Outhwaite, C.; Bhuiyan, L. The modified Poisson-Boltzmann electric double layer capacitance for point ions at low temperatures. J. Electroanal. Chem. 2007. 609, 51.

[50] Jho, Y.S.; Park, G.; Chang, C.S.; Pincus, P.A.; Kim, M.W. Effects of dielectric discontinuities on two charged plates. Phys. Rev. E 2007. 76, 011920.

[51] Jho, Y.S.; Kanduč, M.; Naji, A.; Podgornik, R.; Kim, M.W.; Pincus, P.A. Strong-coupling electrostatics in the presence of dielectric inhomogeneities. Phys. Rev. Lett. 2008. 101, 188101.

[52] Boda, D.; Gillespie, D.; Nonner, W.; Henderson, D.; Eisenberg, B. Computing induced charges in inhomogeneous dielectric media: Application in a Monte Carlo simulation of complex ionic systems. Phys. Rev. E 2004. 69, 046702.

[53] Boda, D.; Valiskó, M.; Eisenberg, B.; Nonner, W.; Henderson, D.; Gillespie, D. The effect of protein dielectric coefficient on the ionic selectivity of a calcium channel. J. Chem. Phys. 2006. 125, 034901.

[54] Croxton, T.; D. A, M.; Patey, G.N.; Torrie, G.M.; Valleau, J.P. Ionic solution near an uncharged surface with image forces. Can. J. Chem. 1981. 59, 1998.

[55] Bratko, D.; Jönsson, B.; Wennerström, H. Electrical double layer interactions with image charges. Chem. Phys. Lett. 1986. 128, 449. 
[56] Linse, P. Image charge effects in spherical symmetry with applications to micellar systems. $J$. Pyhs. Chem. 1986. 90, 6821.

[57] Messina, R. Image charges in spherical geometry: Application to colloidal systems. J. Chem. Phys. 2002. 117, 11062.

[58] Alawneh, M.; Henderson, D. Monte Carlo simulation of the double layer at an electrode including the effect of a dielectric boundary. Mol. Sim. 2007. 33, 541.

[59] Alawneh, M.; Henderson, D.; Outhwaite, C.W.; Bhuiyan, L.B. The effect of dielectric polarization of the electrode on anomalous temperature effects in the electrical double layer. Mol. Sim. 2008. 34, 501.

[60] Hatlo, M.M.; Lue, L. The role of image charges in the interactions between colloidal particles. Soft Matter 2008. 4, 1582.

[61] Reščič, J.; Linse, P. Potential of mean force between charged colloids: Effect of dielectric discontinuities. J. Chem. Phys. 2008. 129, 114505.

[62] Wang, Z.Y.; Ma, Y.Q. Monte Carlo determination of mixed electrolytes next to a planar dielectric interface with different surface charge distributions. J. Chem. Phys. 2009. 131, 244715 .

[63] Wang, Z.Y.; Ma, Y.Q. Impact of head group charges, ionic sizes, and dielectric images on charge inversion: A Monte Carlo simulation study. J. Phys. Chem. B 2010. 114, 13386.

[64] Holovko, M.; Kapko, V.; Henderson, D.; Boda, D. On the influence of ionic association on the capacitance of an electrical double layer. Chem. Phys. Lett. 2001. 341, 363.

[65] Bhuiyan, L.B.; Outhwaite, C.W.; Henderson, D. A modified Poisson-Boltzmann analysis of the capacitance behavior of the electric double layer at low temperatures. J. Chem. Phys. 2005. 123, 034704. 
[66] Caprio, D.D.; Valiskó, M.; Holovko, M.; Boda, D. Anomalous temperature dependence of the differential capacitance in valence asymmetric electrolytes. Comparison of Monte Carlo simulation results and the field theoretical approach. Mol. Phys. 2006. 104, 3777.

[67] Boda, D.; Chan, K.Y.; Henderson, D. Monte Carlo simulation of an ion-dipole mixture as a model of an electrical double layer. J. Chem. Phys. 1998. 109, 7362.

[68] Nagy, T.; Gillespie, D.; Boda, D. Neumann and Dirichlet boundary conditions for planar double layers in the constant charge and constant voltage ensembles 2010. In preparation.

[69] Barthel, J.; Buchner, R.; Münsterer, M. DECHEMA Chemistry Data Series, vol. 12. Frankfurt a.M.: DECHEMA, 1995.

[70] Fawcett, W.R.; Tikanen, A.C. Role of solvent permittivity in estimation of electrolyte activity coefficients on the basis of the mean spherical approximation. J. Phys. Chem. 1996. 100, 4251.

[71] Vincze, J.; Valiskó, M.; Boda, D. The nonmonotonic concentration dependence of the mean activity coefficient of electrolytes is a result of a balance between solvation and ion-ion correlations. J. Chem. Phys. 2010. 133, 154507. 


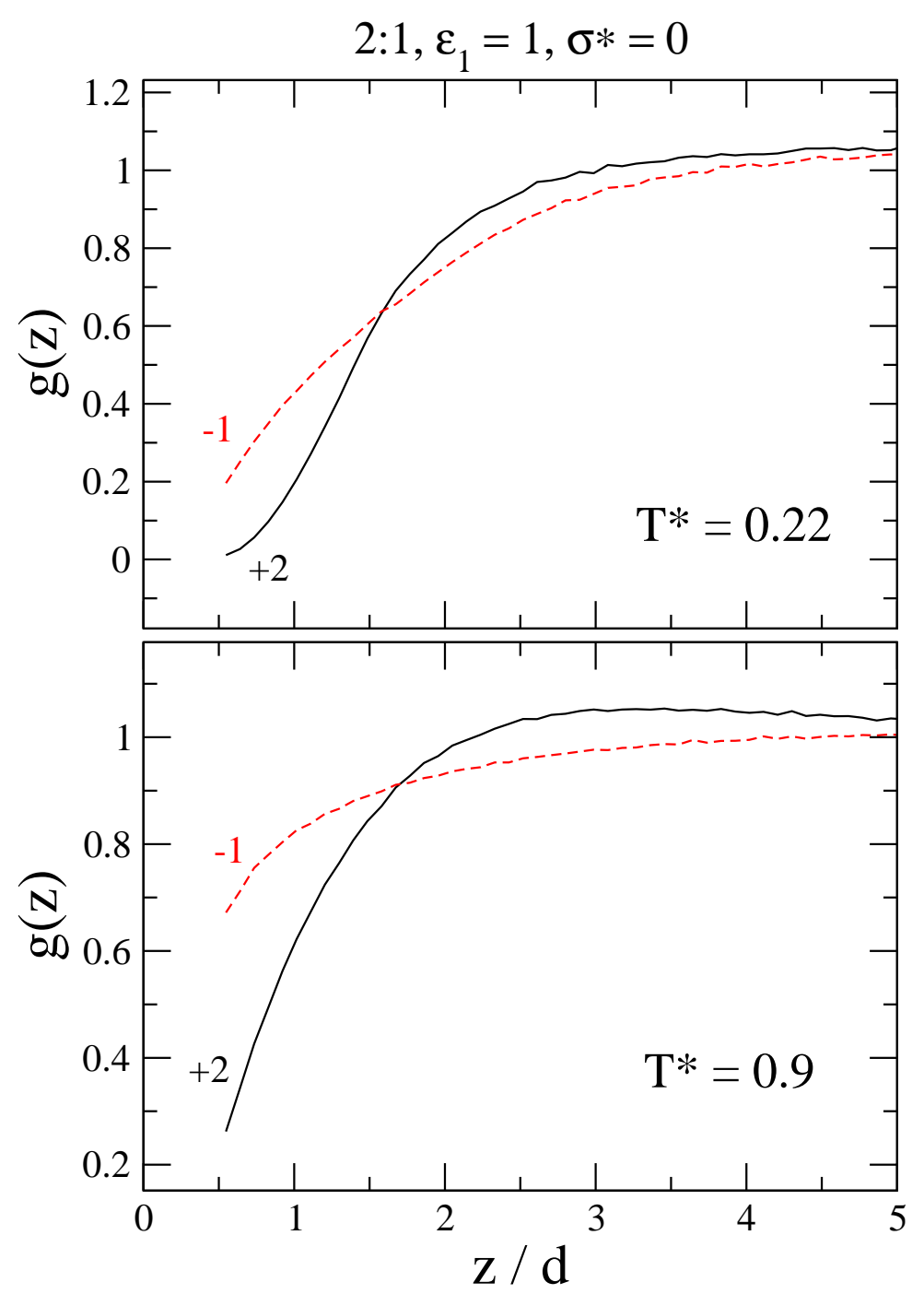

Figure 1: Normalized density profiles for a 2:1 electrolyte near an air/solution interface $\left(\epsilon_{1}=1\right)$ for $T^{*}=0.22$ (top panel) and $T^{*}=0.9$ (bottom panel). The electrode is uncharged $\left(\sigma^{*}=0\right)$. 


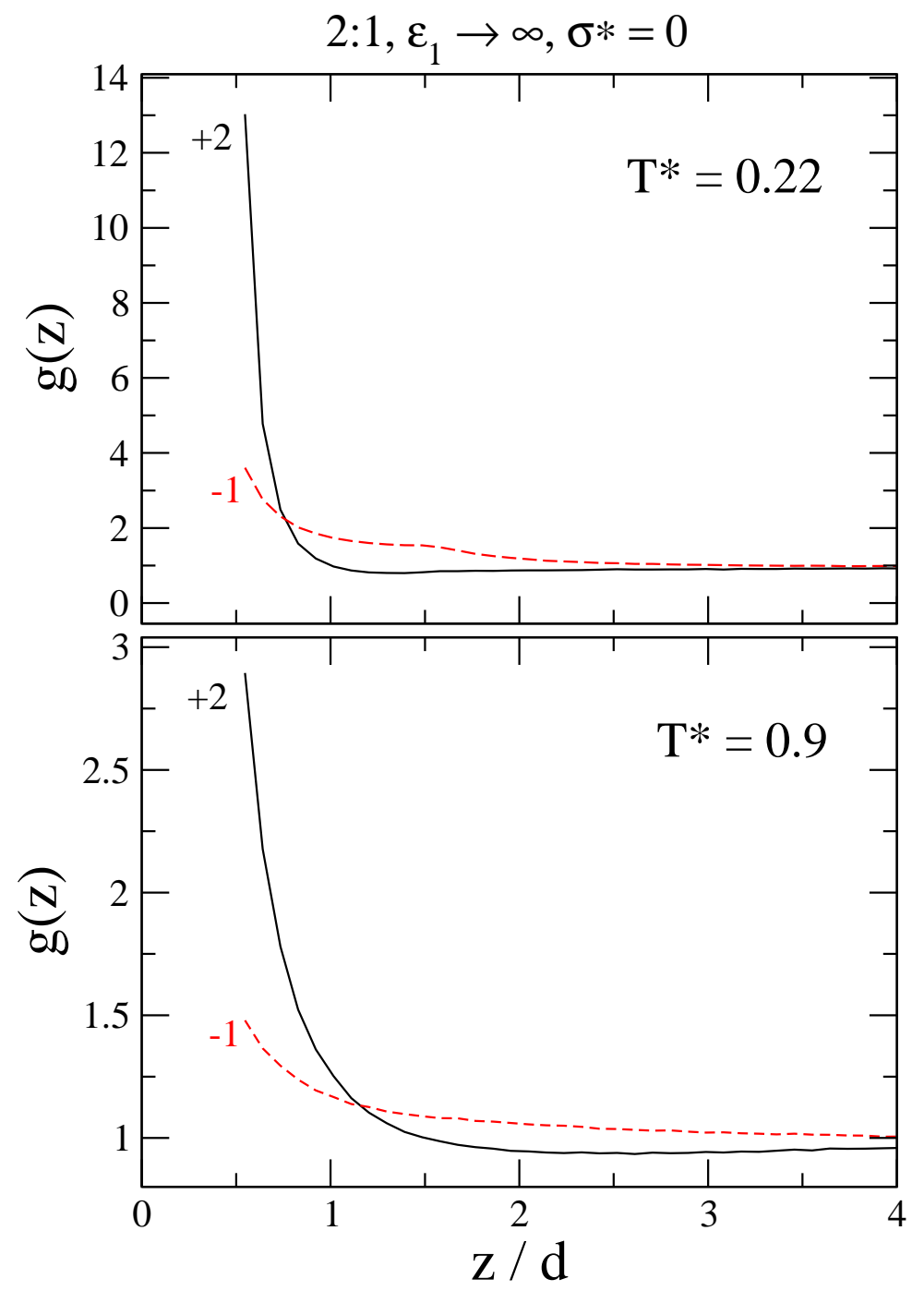

Figure 2: Normalized density profiles for a 2:1 electrolyte near a metal/solution interface $\left(\epsilon_{1} \rightarrow \infty\right)$ for $T^{*}=0.22$ (top panel) and $T^{*}=0.9$ (bottom panel). The electrode is uncharged $\left(\sigma^{*}=0\right)$. 


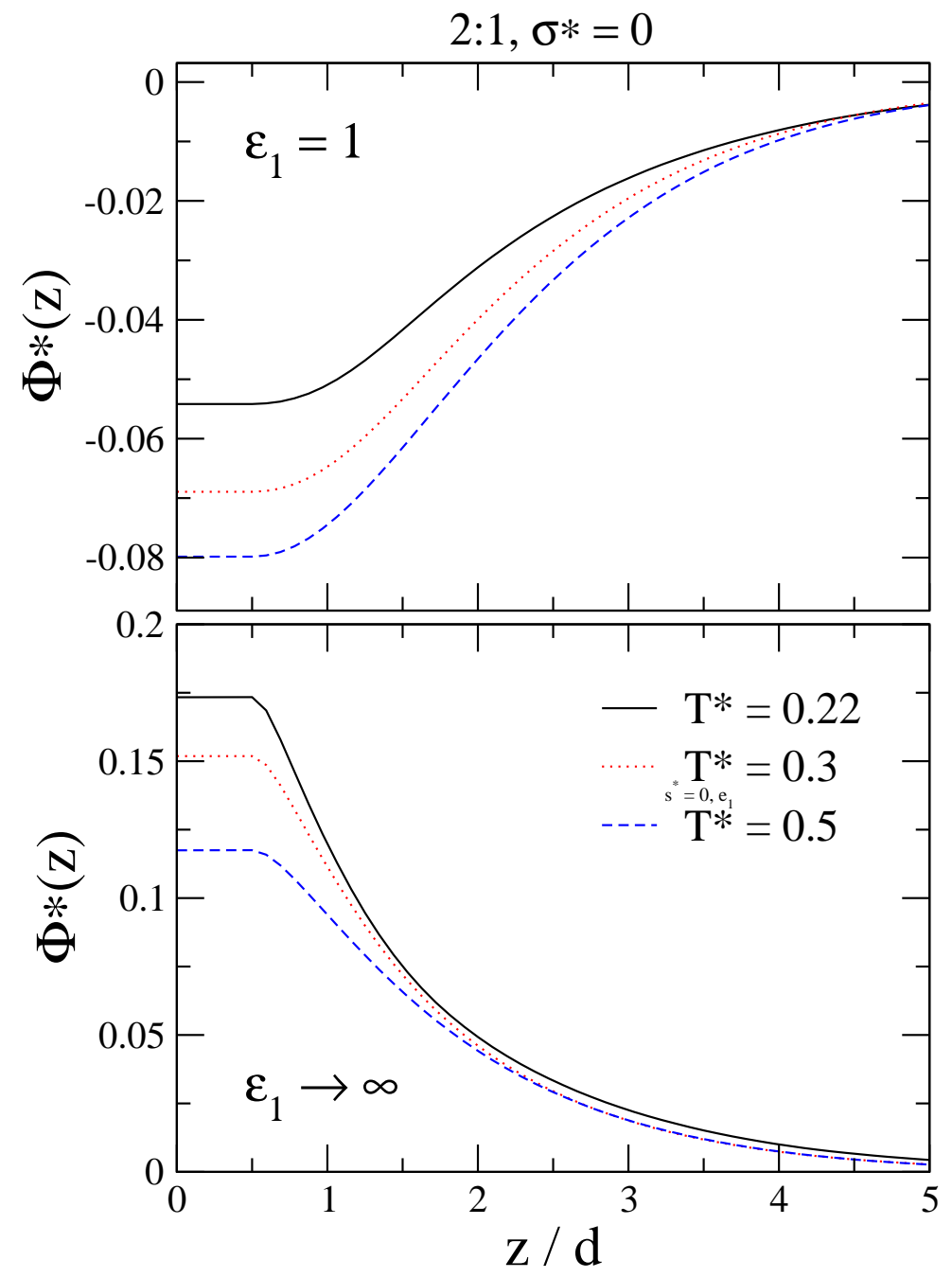

Figure 3: The electrical potential profiles for a 2:1 electrolyte near an air/solution interface $\left(\epsilon_{1}=1\right.$, top panel) and near a metal/solution interface $\left(\epsilon_{1} \rightarrow \infty\right.$, bottom panel) for three different reduced temperatures $\left(\sigma^{*}=0\right)$. 


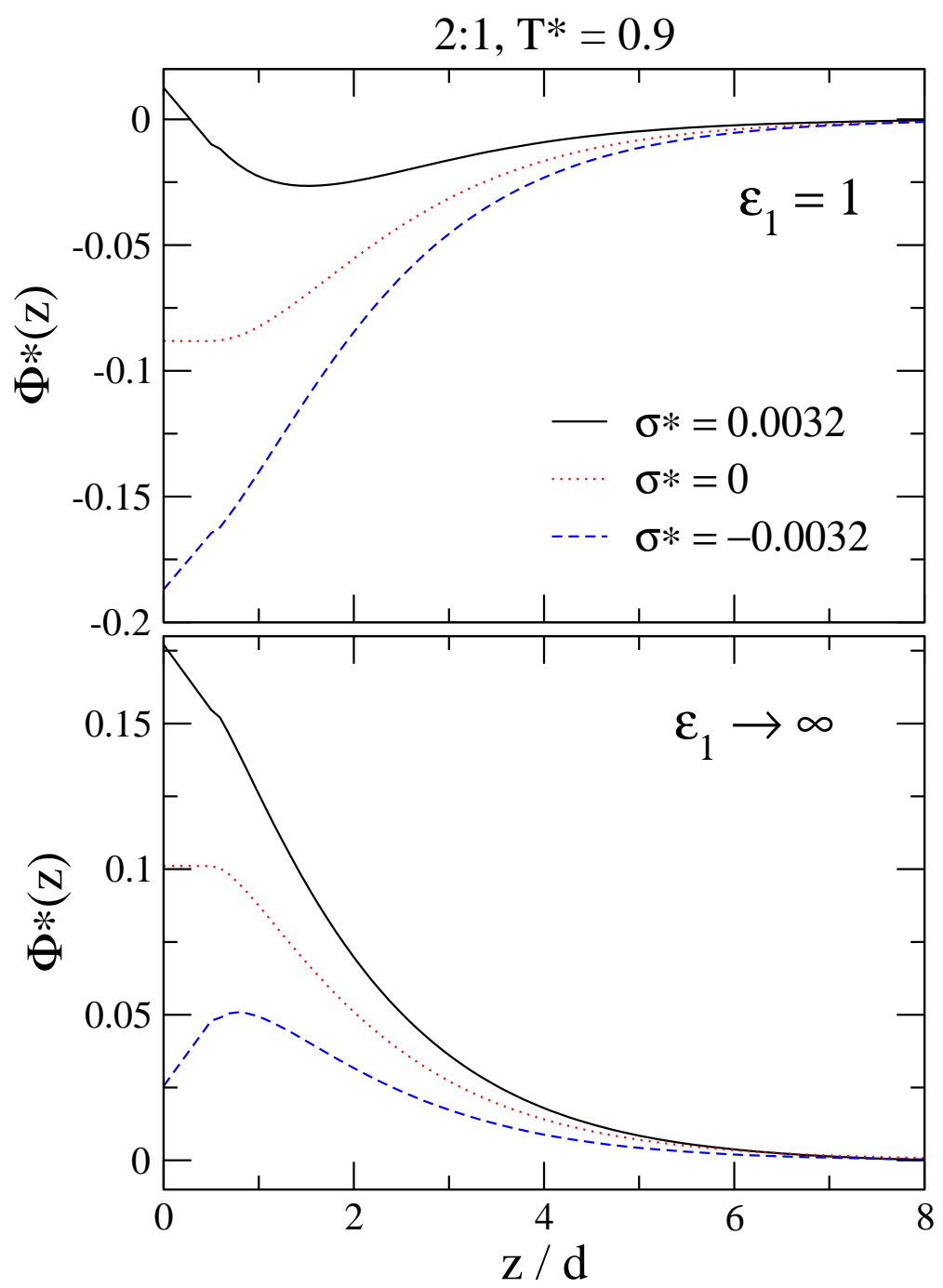

Figure 4: The electrical potential profiles for a 2:1 electrolyte near an air/solution interface $\left(\epsilon_{1}=1\right.$, top panel) and near a metal/solution interface $\left(\epsilon_{1} \rightarrow \infty\right.$, bottom panel) for three different reduced surface charges $\left(T^{*}=0.9\right)$. 


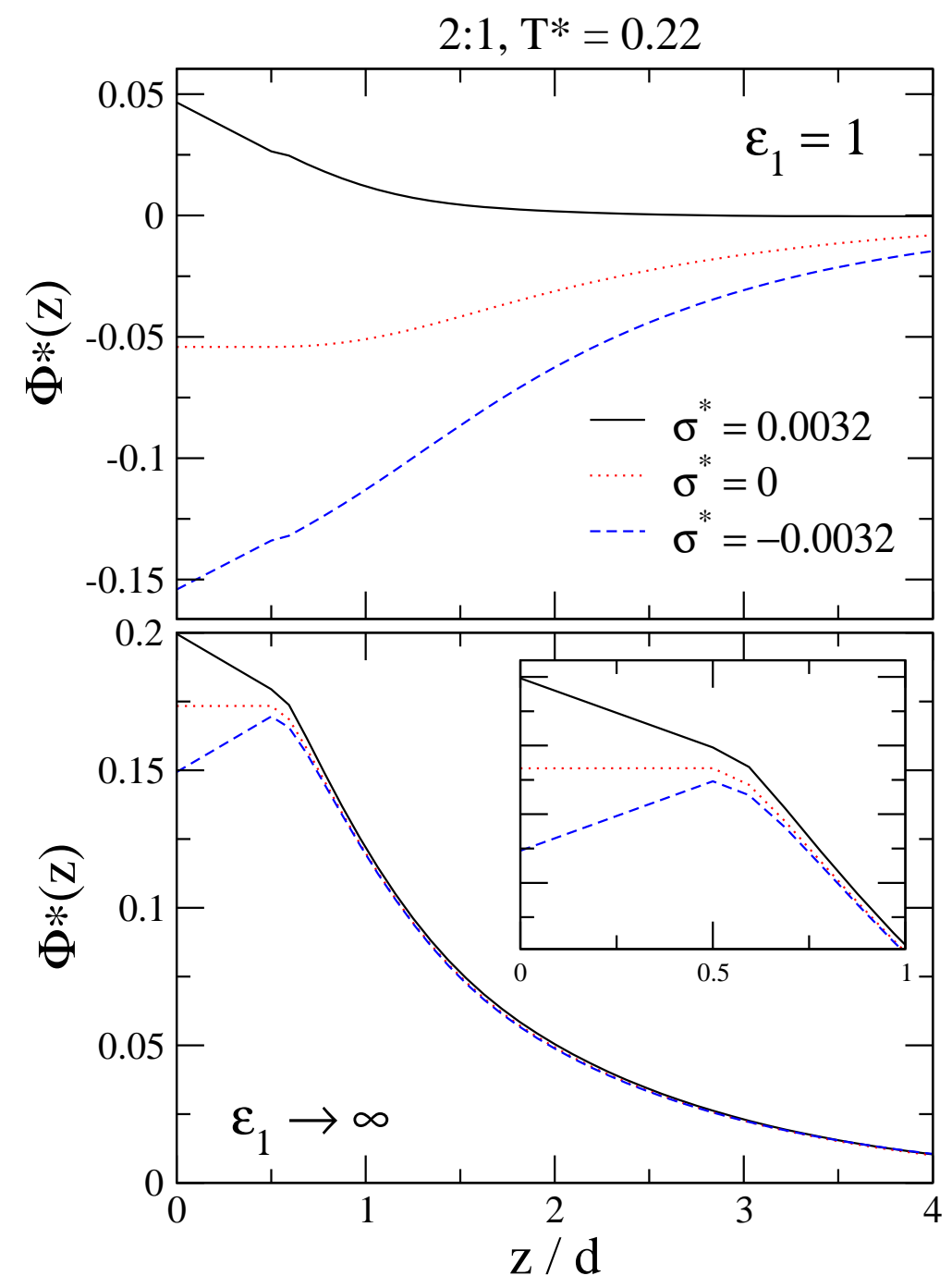

Figure 5: The electrical potential profiles for a 2:1 electrolyte near an air/solution interface $\left(\epsilon_{1}=1\right.$, top panel) and near a metal/solution interface $\left(\epsilon_{1} \rightarrow \infty\right.$, bottom panel) for three different reduced surface charges $\left(T^{*}=0.22\right)$. The inset shows the curves close to the surface. 

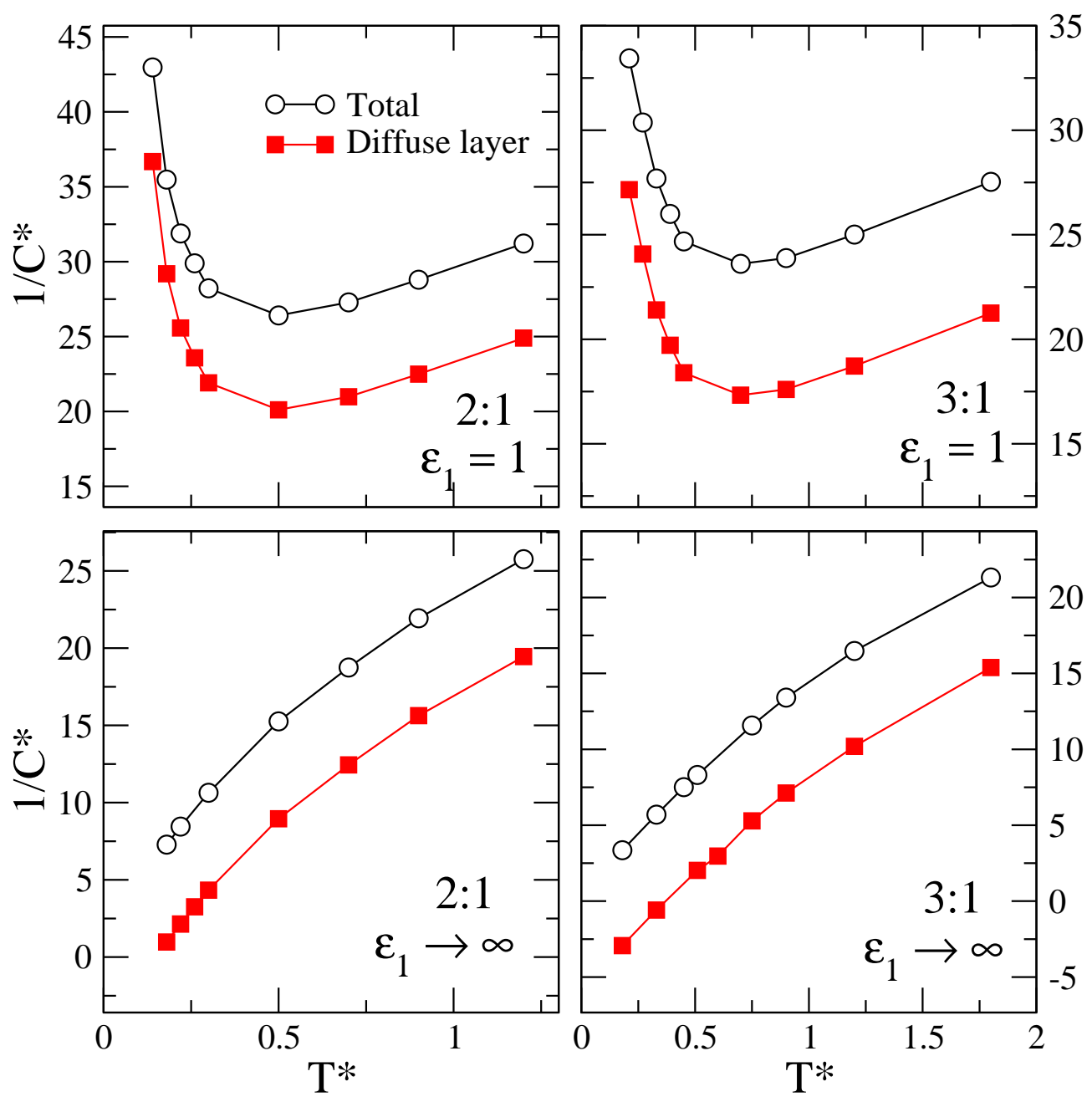

Figure 6: The inverse capacitance as a function of $T^{*}$ for 2:1 (left panels) and 3:1 (right panels) electrolytes near an air/solution interface $\left(\epsilon_{1}=1\right.$, top panels) and near a metal/solution interface $\left(\epsilon_{1} \rightarrow \infty\right.$, bottom panels). The figure shows both the total capacitance $1 / C^{*}$ and its diffuse layer component $1 / C_{\mathrm{D}}^{*}$. 